\title{
Quantum Symmetries
}

Chelsea Walton

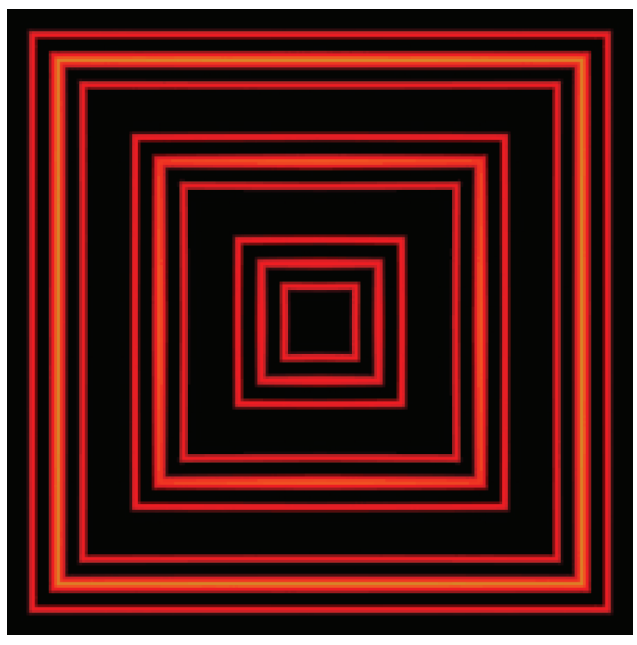

he motivation of this talk is the classical notion of symmetry. Mathematically, a symmetry of an object is an invertible, property-preserving transformation of the given object. Further, a collection of such symmetries forms a group. For instance, consider symmetries of a square-these include reflections, rotations, translations, and also scaling; indeed, the proportions of side-lengths, angles, and orientation of the square are preserved under these invertible transformations.

On the other hand, take the object below:

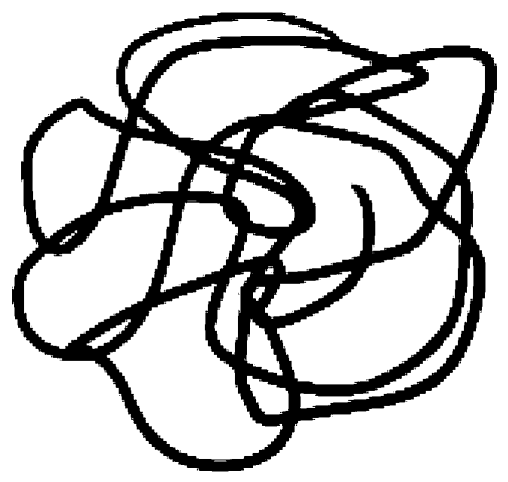

Chelsea Walton is Bloch Brown Assistant Professor of Mathematics at Temple University. Her email address is not 1 aw@temp 1e.edu.

For permission to reprint this article, please contact: reprint-permission@ams.org.

DOI: http://dx.doi.org/10.1090/noti1428
Now we would say that this object does not admit many symmetries, that is, this object is more rigid than the square.

But let us turn our attention to objects that algebraists know and love: algebras! For a fixed algebra $A$, we will study invertible transformations of $A$ (i.e., bijective vector space maps) that preserve the multiplication and unit of $A$. The algebras of interest here are finitely presented, are associative, and are not necessarily commutative.

For example, take $A$ to be $\mathbb{C}[x, y]$, a polynomial ring in two variables over $\mathbb{C}$. This algebra is commutative and admits a myriad of symmetries, including those induced by the following linear transformations:

$$
\left\{\begin{array}{l}
x \stackrel{g \cdot}{\mapsto} a x+c y, \\
y \stackrel{g \cdot}{\mapsto} b x+d y,
\end{array} \text { for } g=\left(\begin{array}{ll}
a & b \\
c & d
\end{array}\right) \in G L_{2}(\mathbb{C}) .\right.
$$

In particular, if we realize $\mathbb{C}[x, y]$ as the quotient of the free algebra $\mathbb{C}\langle x, y\rangle$ by the ideal $(x y-y x)$, it is easy to check that any action of the group element $g$ as above preserves both $\mathbb{C}\langle x, y\rangle$ and the relation space $\mathbb{C}(x y-y x)$ of $\mathbb{C}[x, y]$.

Next, let us take a noncommutative algebra that has many of the same (ring-theoretic and homological) properties of $\mathbb{C}[x, y]$ : the $q$-polynomial ring

$$
\mathbb{C}_{q}[x, y]:=\mathbb{C}\langle x, y\rangle /(x y-q y x)
$$

for $q \in \mathbb{C}^{\times}$. Using the linear transformation above, we obtain:

$$
\begin{aligned}
g \cdot( & (x y-q y x) \\
= & (g \cdot x)(g \cdot y)-q(g \cdot y)(g \cdot x) \\
= & (1-q)(a b) x^{2}+(a d-q b c) x y \\
& -\left(a d-q^{-1} b c\right) q y x+(1-q)(c d) y^{2} .
\end{aligned}
$$

Thus, as soon as we move away from $q=1$, the number of $g$-actions that preserve the relation space of $\mathbb{C}_{q}[x, y]$ decreases. In fact, for $q \neq \pm 1$, the collection of these actions are limited to merely the diagonal ones. So, the algebra $\mathbb{C}_{q}[x, y]$ for $q \neq 1$ is more rigid than $\mathbb{C}[x, y]$ in the context of linear group actions.

This phenomenon occurs in general: noncommutative algebras admit fewer group actions than their commutative counterparts. Therefore in order to have a rich theory of We must think beyond group actions! symmetries of noncommutative algebras, we must think beyond group actions! 
American Mathematical Society

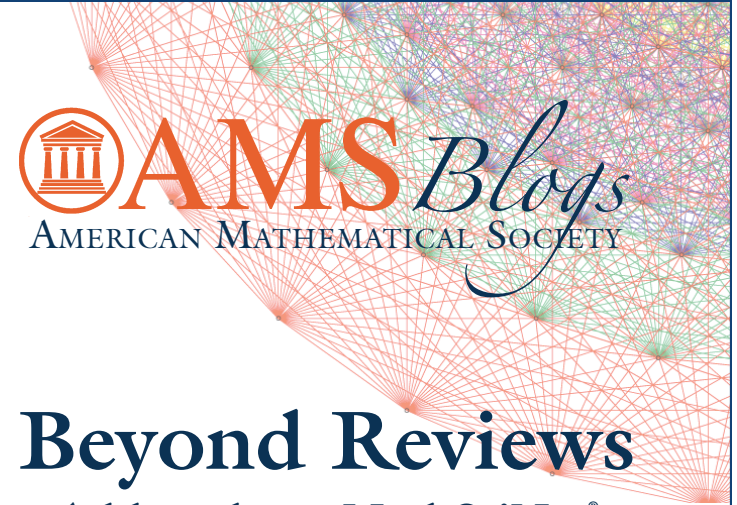

A blog about MathSciNet ${ }^{\circ}$

From the Executive Editor of Mathematical Reviews, Edward Dunne

A blog created to highlight the innovative features of MathSciNet. Updates will include particularly informative reviews and will discuss tips and tricks for navigating MathSciNet, all with the goal of being helpful to users both old and new.

\section{blogs.ams.org/beyondreviews}

AMERICAN MATHEMATICAL SOCIETY

\section{MathSciNet} Mathematical Reviews
Group actions

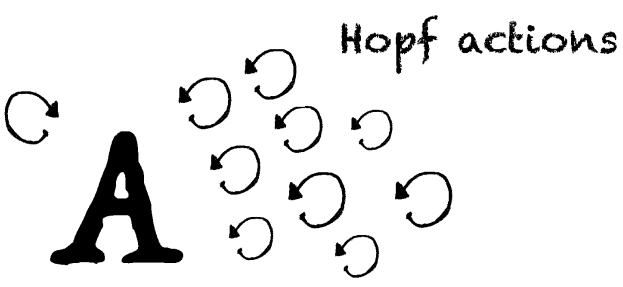

(Noncommulative) Algebra

In the talk, we will discuss quantum symmetries, or (co)actions of Hopf algebras/quantum groups on (noncommutative) algebras.

Everything, from the notion of a Hopf algebra to its (co)actions on algebras, will be defined and illustrated by plenty of examples. We will also discuss various recent results (of mine, with coauthors, and of many others) in this fruitful direction of research.

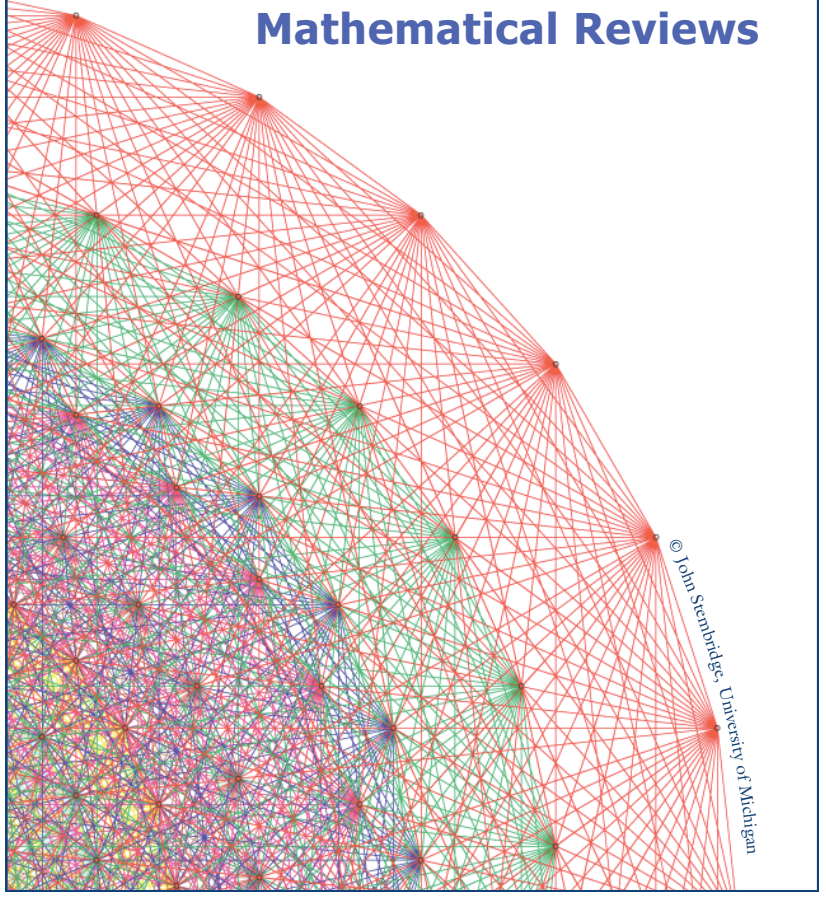

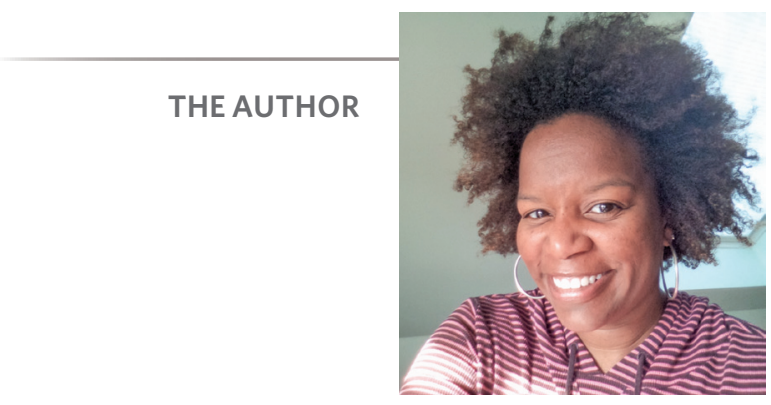

Chelsea Walton 